\title{
Orthorexia vs. theories of healthy eating
}

\author{
Steven Bratman ${ }^{1}$
}

Received: 22 April 2017/Accepted: 5 July 2017 / Published online: 24 July 2017

(C) Springer International Publishing AG 2017

Orthorexia has two stages. The first is innocent, generally laudatory: choosing to eat a healthy diet. The second involves an intensification of that pursuit into an unhealthy obsession. It is only this subsequent stage that implicates pathology. However, when the first step involves adoption of non-standard dietary ideas that appear irrational, unscientific or strange, the label of disordered eating is sometimes applied prematurely.

In my opinion, this is an error with consequences.

In retrospect, it appears that I contributed to this error in my initial writing on orthorexia. This editorial presents an attempt to rectify the confusion I inadvertently contributed to.

\section{Origin story}

When I coined the term orthorexia nervosa 20 years ago, I did not intend to propose a new eating disorder.

At that time, I was a practitioner of "alternative medicine," a medical subculture that includes herbal therapies, acupuncture, dietary supplements, and numerous other non-mainstream methods and theories.

The many schools of alternative medicine derive from a variety of locations in time and place and hold contradictory axioms. Yet, most share a belief that certain food choices are critical to wellness. Conventional medicine would agree, but its recommendations are relatively simple. The dietary theories associated with alternative

This article is part of the topical collection on Orthorexia Nervosa.

Steven Bratman

stevenbratman@gmail.com

Vallejo, California, United States medicine, on the other hand, tend to include numerous highly specific and often deeply challenging recommendations. This can lead to problems.

In the mid-1990s, I came to believe that many of my more diet-focused patients were inadvertently harming themselves psychologically through excessive focus on food. They had reduced the dimensionality of their human lives by assigning excessive meaning and power to what they put in their mouths. Their exuberant pursuit of physical health had spawned a rigid, fearful and self-punishing lifestyle that caused more harm than good.

As an alternative medicine practitioner, I believed first and foremost in "treating the whole person." From this perspective, my patients needed to relax the dietary corset and live a little. This advice was difficult to carry across. My patients viewed healthy eating as a fundamental virtue. How can one lighten up on a virtue? To ask a patient to relax her diet is tantamount to suggesting she embark on a life of crime. I might as well advise, "Go and commit some larceny. Drive drunk a little. It will be good for you."

To get around this I used the therapeutic trick of naming. With the help of a Greek scholar, I coined the term orthorexia nervosa, formed in analogy to anorexia nervosa, but using "ortho," meaning "right," to indicate an obsession with eating the right foods. From then on, whenever a patient would ask me what food she should cut out, I would say, half tongue-in-cheek, "We need to work on your orthorexia." The word turned expectations upside down and opened a pathway to further discussion.

In these discussions, I frequently critiqued the theories of diet that my patients followed. My goal was to loosen the grip these theories held on my patients' minds. I could do this because I was myself an "insider" to those theories and an expert upon them. In the initial essay on orthorexia I published in Yoga Journal in 1997 and the subsequent book length 
treatment Health Food Junkies in 2000, I devoted considerable attention to deconstructing dietary theories as well, still from the perspective of an insider $[1,2]$. I had "permission" to criticize because I spoke the language and understood the viewpoint often even better than my readers did.

The book was also part of my personal journey.

I had originally joined the alternative diet subculture in the 1970s as an earnest young convert to the "back-to-theland" movement. My professional practice of alternative medicine had logically followed. However, over a period of many years I intellectually deconstructed this adherence, through a process that involved direct observation and the composition of more than 20 books and a database on evidence-based evaluation of alternative medicine. By the time Health Food Junkies hit the shelves, I no longer found any alternative dietary theory credible. Nonetheless, I retained an understanding of the beliefs and a sympathy and respect for those who hold them.

In the subsequent decade, my therapeutic trick came to be analyzed in the literature as a possible eating disorder. I was not directly involved in this research, but I followed the publications with surprise and interest. One obvious observation was that those tackling the subject were writing from the outside; they had never lived within the world of alternative eating cultures, and therefore at times seemed to misunderstand them. For example, some writers mistook features of one or another specific dietary theory for a universal characteristic of all orthorexia. Others seemed to view belief in false theories as a sign of disordered thinking, and enthusiasm for these dietary theories as prima facie evidence of disordered eating.

This latter attitude found its way into popular media, where mere veganism or a desire to avoid processed foods was often reduced to orthorexia. In response, alternative medicine communities defended themselves against these caricatures, sometimes denouncing the concept of orthorexia as a construction designed by corporatist forces to encourage people eat unhealthy food.

These developments seemed to me to be dangerous and problematic: The very people who might most benefit from the concept of orthorexia were developing cultural antibodies against it.

In 2016, I responded to these concerns by working with Thomas Dunn to design new formal criteria for orthorexia nervosa that explicitly avoided these errors [3]. In the paper that accompanied the criteria, I did not have space to provide the necessary background information. I provide some of it here.

\section{A brief introduction to theories of healthy eating}

The attempt to treat or prevent illness via diet has a long international history [2, 4]. Many ancient ideas persist today, combined with or altered by more recent thinking.
Some examples are veganism, macrobiotics, paleo and advice to avoid "rich" or fatty foods. Other approaches have been entirely invented in recent decades or centuries, such as food combining, acid/alkaline theory, food sensitivities and an entire class of post-industrial ideas typified by the current Clean Eating movement.

Macrobiotics, which originated in nineteenth century Japan, seeks to "balance yin and yang" and characterizes all foods on a yin/yang spectrum. The theory contains elements common to the background eating cultures of many East Asian countries, such as a characterization of all raw vegetables as unhealthy, but adds numerous specific details down to the health implications of proper vegetable slicing.

Raw Foods Veganism, in contrast, idealizes raw vegetables as superior to all other foods, and quite literally characterizes the cooking stove as a major source of human illness.

Paleo Diet theory can be detected in the Avoid Grains theory of ancient Taoist China [5], but arose independently in the West in the 1990s. Utilizing philosophical concepts and weak science, it counsels avoiding foods that came into use after the invention of agriculture.

Dietary supplement theories arose in naturopathic medicine after being condemned by the original generation of naturopathic physicians. It eventually came to include numerous substances that can replace ordinary diet, but are only food-like. These are seen variously to prevent cancer, enhance sports performance and increase longevity.

There is a broad class of diet theories of ancient and modern vintage foreground concepts of food purity. Indian Ayurvedic medicine includes one version of this. Another version arose post-industrial revolution, and currently involves eschewing "refined" and "processed" foods, food additives, GMOs and antibiotic-fed animal products. In these approaches, foods themselves are conceptualized as "pure" and "impure." As a distinct but related issue, choice of pure food is seen to yield a pure body. Fasting or "detoxing" is commonly added to enhance the sense of internal cleanliness.

At the opposite extreme are novel dietary theories based on a single recent source. The popular Blood Type Diet is an example. This trademarked method provides precise dietary recommendations for each major blood type, and although the recommendations are complex they originate in a single book published in 1996 [6].

It is important to state that most alternative healthy eating beliefs can be adhered to safely; most followers of alternative diet theories do not have orthorexia. Bill Clinton is a vegan and Jeb Bush follows a paleo diet. Gandhi was often a raw foodist, and followed a variety of other extreme dietary beliefs. The development of orthorexia is a separate stage. 


\section{When does health foodism become pathological?}

Interest in healthy eating does not become pathological until a further progression takes place. In this second stage, obsessive thinking, compulsive behavior, self-punishment, escalating restriction and all the other dynamics of conventional eating disorders begin to take hold.

In general, the more complex or severe the theory, the more material it provides for orthorexic acceleration. Theories based on purity of food and body may lead to (or derive from) struggles with a sense of personal impurity, uncleanness and shame. Theories based on balance can emphasize struggles with control. Those based on concepts of food sensitivities enhance fear; morally based theories lead to superiority to others, and so on.

Note that it is quite commonly observed that once people develop orthorexia, they may quite suddenly switch from one theory to another, even if the principles of the new theory contradict those of the former. This process of switching can be very dramatic, and helps illustrate the distinction between the underlying theory and the supervening pathology.

\section{Attacking dietary theories}

Most alternative dietary theories are largely unscientific; many are pseudoscientific, anti-scientific or just plain bizarre. It is therefore natural for mainstream authorities to attack these theories. While such an approach can be an essential educative action if handled carefully, it may cause harm if deployed in a reflexive, unsympathetic manner.

Most non-mainstream dietary theories are endorsed by famous and influential people (including apparently authoritative medical doctors) and supported by large eating communities with extensive literature. Furthermore, many of these theories explicitly view mainstream medicine as a part of a deeply corrupted corporate culture that conspires to seek profit at the expense of health; other theories simply view the conventional view as erroneous. When mainstream authorities attack the theory, this only confirms expectations. Members of the attacked diet community adopt a defensive posture, and those most deeply involved become walled off and more difficult to reach.

Critique of alternative diet can sometimes be an appropriate therapeutic step in individual counseling once trust has been established. However, it makes a poor opening salvo. Above all, such critique should not be written into the definition of orthorexia, because that will inspire a detailed defensive literature (as has already begun.)
Perhaps, it should be noted that conventional dietary theory too is subject to fads, fashion and overt self-interest. The universal 1960s era recommendation to avoid eggs and consume margarine in lieu of butter is an obvious example of an erroneous enthusiasm, and the only recently debunked low-fat diet hypothesis that was initially sponsored by the sugar industry is a recently unearthed scandal [7]. These problems are well known in the alternative diet world and tend to reduce the credibility of the mainstream when it critiques alternative dietary theories.

In any case, the unscientific nature of the dietary theory is inessential to the condition. In Norway, people are said to develop orthorexia by following the general dietary guidelines of the Health Ministry to an extreme degree. In the USA, I commonly hear from parents who complain that their children developed orthorexia after taking healthy eating class at school. At least a few people have told me they became orthorexic after their cardiologists recommended the rather extreme but conventionally accepted Ornish diet.

It is not the theory itself: it is the response to the theory that constitutes orthorexia.

There is a strong philosophical reason to avoid pathologizing alternative theories of diet as well. All of us come to believe what we believe based on the literature we read, the experts we trust and the communities we belong to. A perfectly sane person may believe something that is unpopular, unscientific or just plain wrong. To pathologize beliefs rather than countering them is akin to requiring thought orthodoxy. This has no place in civil society.

\section{Healthy eating theories and orthorexia criteria}

The sample criteria for orthorexia I proposed with Thomas Dunn explicitly separate the specifics of health theory from its intensification. Indeed, I was largely motivated to enter the criteria-writing world because I thought that existing approaches to diagnosing orthorexia conflated the two steps.

Note that these proposed criteria were explicitly designed to build upon and supercede the so-called Moroze Criteria, proposed in a previous paper in which the primary listed author is listed as R Moroze, but in fact was primarily authored by Dunn [8].

The Moroze Criteria begin with a section A describing "obsessional preoccupation with eating 'healthy foods' regarding the quality and composition of meals." The description of healthy foods in the A subsections fixate on a single, currently popular theory of healthy eating and therefore would become dated as such fashions change. In my opinion, this is a fundamental error and it is changed in the revised criteria. In addition, certain inessential and 
uncommon secondary features of orthorexia are included in the subsections, such as spending excessive money on food choices, and these are modified.

Additional sections describe when the condition becomes impairing and attempt to separate it from other diagnoses, and, in modified form, are largely included in the Dunn and Bratman criteria. However, the Moroze criteria do not include an attempt to distinguish ON from traditional eating disorders based on the presence or absence of thinking about weight. A carve out regarding this issue has been added to the revised criteria.

Another set of interesting criteria was proposed by Barthels [9], consisting of five sections that succinctly describe key elements of orthorexia, including preoccupation, anxiety, overvalued health ideas, impairment and, as with the Dunn and Bratman criteria, a carve out for ideation about weight. I find these to be quite promising, and believe that they could form the basis for primary criteria. However, I have concerns about the use of the term "ritualized" in section $\mathrm{C} 2$, which currently reads, "Ritualized preoccupation with buying, preparing and consuming foods, which is not due to culinary reasons but stems from overvalued ideas."

Ritualization certainly does occur in orthorexia, as in other EDs, but to a considerable extent this is derived from the underlying theory. Many dietary theories prescribe details of food preparation for pseudoscientific reasons; therefore, performance of rituals by followers is a result of adherence to theory rather than expression of a personal pathology. People with highly simplified diets may also seem to follow a ritual when they eat and prepare food, but among those who eat only a handful of different foods, and only for their health value rather than taste, a high degree of repetition is natural.

In general, the central focus in orthorexia is on belief and ideology; the theory exerts an extreme force and prominence, and behavior flows from belief (at least initially). Therefore, if the Barthels Criteria are used, I would recommend changing the cited text to read: "Preoccupation with buying, preparing and consuming food believed to be healthy, which is not due to culinary reasons but stems from overvalued ideas about the healthy-promoting and healthdamaging properties of certain foods of food classes."

As noted, both the Barthels and the Dunn and Bratman criteria indicate that in $\mathrm{ON}$ weight loss is not a primary motivation. While this was certainly true in my original description of orthorexia, many clinicians have pointed out that this distinction may be problematic in real life, as for many people ideation about "healthiness" has become closely intertwined with ideas about body weight and body fat percentage. This remains a thorny issue that will need further investigation.

I do wish to state that I am not attached to any particular criteria for orthorexia, including my own. However, I believe that any proposed criteria and associated survey tools should reflect the distinctions described in this essay. Criteria should not reference specific elements of a currently popular dietary theory because these will change over time. Criteria and survey tools must also distinguish between exceptional enthusiasm for eating healthy food and the subsequent intensification into obsessive thinking and compulsive behavior. Finally, the "correctness" or "incorrectness" of the underlying theory should not be made an issue for all the reasons described above.

\section{Orthorexia: diffusion and development}

In my initial observations in the 1990s, orthorexia arose primarily in alternative medicine subcultures where specific healthy food diets are espoused. Subsequently, interest in healthy eating has pervaded popular culture. Conversation about and photography of health food fills social media, healthy eating promotion has been taken up by governmental authorities, and corporate food marketing departments now brand their products as healthy rather than low calorie. Because interest in healthy food is a necessary prerequisite for developing orthorexia, this broadening of interest has facilitated increased prevalence of the subsequent condition.

In addition, there has been a melding of several distinct concepts: losing weight, improving health and enhancing healthy appearance. The term fitness, which once meant "an ability to walk up hills without getting short of breath" now also describes the "fit" body type. Low calorie foods are seen as somewhat identical to healthy foods. Being healthy does not only mean reduced risk of cancer, it also implies "glowing skin."

All of the above changes, as well as evolution in the definition of "anorexia nervosa," have brought orthorexia closer to anorexia.

Finally, exercise addiction is now increasingly found in association with orthorexia, something that was not present when I originally defined it. (As a matter of historical oddity, it appears that in Sweden orthorexia has come to mean exercise addiction [10]).

\section{Conclusion}

It has been interesting to observe the subsequent development of an idea that 1 rather casually launched 20 years ago. From the widespread utilization of the non-existent Bratman Orthorexia Test through the spread of dozens of "orexias" including bigorexia and fitorexia, to the peculiar meaning evolution in Sweden, this has been a fascinating demonstration of the fluidity of ideas. I look forward to 
future developments. Of most importance, it remains to be discovered whether there proves to be clinical utility to defining a distinct ED-labeled orthorexia, or whether such a distinction becomes moot in light of subsequent evolution of existing EDs.

Level of evidence Level V, editorial.

\section{Compliance with ethical standards}

Conflict of interest I have no conflict of interests regarding this publication.

Ethical approval This article does not contain any studies with human participants performed by any of the authors.

\section{References}

1. Bratman S (1997) Health food junkie. Yoga J September/October: $42-50$

2. Bratman S, Knight D (2000) Health food junkies: orthorexia nervosa: overcoming the obsession with healthful eating. Broadway, New York
3. Dunn TM, Bratman S (2016) On orthorexia nervosa: a review of the literature and proposed diagnostic criteria. Eat Behav 21:11-17. doi:10.1016/j.eatbeh.2015.12.006

4. Bratman S (1997) The alternative medicine sourcebook: a realistic evaluation of alternative healing methods, 1 st edn. Lowell House, Los Angeles

5. Bigu_(grain_avoidance) https://en.wikipedia.org/wiki/Bigu_ (grain_avoidance). Accessed 10 June 2017

6. Adamo P, Whitney C (1996) Eat right 4 your type. Putnams' Sons, New York

7. Kearns CE, Schmidt LA, Glantz SA, Sugar Industry and Coronary Heart Disease Research (2016) A historical analysis of internal industry documents. JAMA Intern Med 176(11):1680-1685. doi:10. 1001/jamainternmed.2016.5394

8. Moroze RM, Dunn TM, Holland JC, Yager J, Weintraub P (2015) Microthinking about micronutrients: a case of transition from obsessions about healthy eating to near-fatal "orthorexia nervosa" and proposed diagnostic criteria. Psychosomatics 56:397-403. doi:10.1016/j.psym.2014.03.003

9. Barthels F, Meyer F, Pietrowsky R (2015) Orthorexic eating behavior. A new type of disordered eating. Ernährungs Umschau 62:156-161. doi:10.4455/eu.2015.029

10. Håman L, Barker-Ruchti N, Patriksson G, Lindgren EC (2015) Orthorexia nervosa: an integrative literature review of a lifestyle syndrome. Int J Qual Stud Health Wellbeing 10:26799. doi:10. 3402/qhw.v10.26799 